\title{
PGRSS de um Hospital Público de Florianópolis: Atendimento aos Requesitos Legais ou Responsabilidade Social Corporativa da Instituição?
}

\author{
Eliane Puperi Alves da Silveira ${ }^{1}$ \\ Janete Zanchin'2 \\ Universidade do Sul de Santa Catarina
}

\section{RESUMO}

O estudo tem como objetivo geral compreender o programa de geração de resíduos de serviços de saúde de um hospital público da região de Florianópolis, verificando se o cumprimento do mesmo se dá apenas por questões legais ou se, além disso existe, de fato, responsabilidade social corporativa por parte da instituição, com real preocupação com a comunidade em que está inserida e com o meio ambiente. Quanto aos objetivos da pesquisa, primeiramente, realizou-se uma pesquisa exploratória, seguida de uma descritiva, nesta última coletou-se dados junto aos gestores/colaboradores da instituição, além de análise de dados indicadores da instituição. Entre os resultados obtidos, percebeu-se que, apesar do programa ter sido criado em virtude dos requisitos legais, já existe certa preocupação com a geração de resíduos e com o impacto ambiental provocado por ele nos colaboradores e gestores do hospital.

Palavras-chave: Plano de Gerenciamento de Resíduos de Serviços de Saúde; PGRSS; Resíduos Hospitalares; Requisitos Legais; Responsabilidade Social Corporativa.

\section{ABSTRACT}

The objective of the study is to understand the health services waste generation program of a public hospital in the region of Florianópolis, verifying whether compliance is only for legal reasons or if, in addition, there is, in fact, social responsibility of the institution, with real concern for the community in which it is inserted and with the environment. Regarding the objectives of the research, first, an exploratory research was carried out, followed by a descriptive one, in the latter one collected data with the managers / collaborators of the institution, in addition to analyzing the institution's indicator data. Among the results obtained, it was noticed that, although the program was created due to the legal requirements, there is already some concern about the generation of waste and the environmental impact caused by it in the employees and managers of the hospital.

Keywords: Waste Management Plan for Waste Resource Management; PGRSS; Hospital Waste; Legal Requirements; Corporate Social Responsibility.

\section{RESUMEN}

El estudio tiene como objetivo general comprender el programa de generación de residuos de servicios de salud de un hospital público de la región de Florianópolis, verificando si el cumplimiento del mismo se da sólo por cuestiones legales o si, además, existe, de hecho, responsabilidad social corporativa por parte de la institución, con real preocupación con la comunidad en que está inserta y con el medio ambiente. En cuanto a los objetivos de la investigación, primero, se realizó una investigación exploratoria, seguida de una descriptiva, en esta última se recogió datos junto a los gestores / colaboradores de la institución, además de análisis de datos indicadores de la institución. Entre los resultados obtenidos, se percibió que, a pesar de que el programa fue creado en virtud de los requisitos legales, ya existe cierta preocupación con la generación de residuos y con el impacto ambiental provocado por él en los colaboradores y gestores del hospital.

Palabras clave: Plan de Gestión de Residuos de Servicios de Salud; PGRSS; Residuos Hospitalarios; Requisitos legales; Responsabilidad Social Corporativa.

\footnotetext{
${ }^{1}$ Pós Graduada em Marketing Esportivo Universidade do Sul de Santa Catarina - puperi.lili@gmail.com

${ }^{2}$ Mestre em Administração(UFSC)-Docente do Departamento de Ciências Sociais Aplicada - Universidade do Sul de Santa Catarina - janete.zanchin@unisul.br
} 


\section{Introdução}

Desde a revolução industrial, a sociedade presencia um grande crescimento das indústrias de bens e serviços, gerando grandes transformações socioeconômicas e ambientais. A sociedade atual testemunha grandes alterações no meio ambiente, que fez com que a partir da década de 1960 as pessoas passassem a refletir sobre as consequências do desenvolvimento das atividades econômicas industriais, buscando formas de impor limites às indústrias, para que as mesmas não causassem danos irreparáveis aos recursos físicos e humanos da Terra (Sanches, 2000). Esse tema ganhou destaque nos últimos anos, interferindo na concepção das estratégias empresariais (Kelm, da Silva Reinz \& Allebrandt, 2014) e vem sendo observada como estratégia para melhoria no desempenho financeiro, resposta a pressões institucionais, discurso de marketing ou atividade pós-lucro (Irigaray, Vergara \& Santos, 2013). A preocupação ambiental torna o gerenciamento de resíduos um processo de extrema importância na preservação do meio ambiente.

Os resíduos gerados pelos serviços de saúde representam um problema complexo por envolver aspectos éticos e técnicos, repercutindo diretamente nos profissionais envolvidos no processo, nas organizações, bem como no meio ambiente. O manejo dos resíduos compreende uma série de processos que se iniciam antes da aquisição dos bens de consumo ampliando-se até o seu destino final. A ideia de gestão integrada de resíduos incluiu a minimização da geração destes, bem como introduzir uma nova cultura de trabalho, contemplando atividades como o manejo adequado dos resíduos, conscientização ambiental, educação continuada e saúde do trabalhador.

Nesse sentido, há muitas organizações públicas e privadas que já praticam o descarte de seus produtos de forma responsável. Uma dessas organizações é um hospital da rede pública do Estado de Santa Catarina com unidades espalhadas estrategicamente pelo estado inteiro, que preferiu não ter seu nome divulgado. A prioridade na gestão de resíduos deste hospital é a não geração, redução, reutilização, reciclagem, tratamento dos resíduos sólidos e disposição final ambientalmente adequada dos rejeitos, refletindo desta forma os valores da Instituição e sua política de qualidade. Diante do exposto, surge a pergunta norteadora deste artigo: as práticas de gestão de um hospital da rede pública de Florianópolis, juntamente com o seu Programa de Gerenciamento de Resíduos existe em virtude da exigência e fiscalização da legislação vigente, ou ele é baseado na responsabilidade socioambiental, não só da instituição, bem como de seus colaboradores, ou comtempla as duas perspectivas?

A metodologia empregada no desenvolvimento do estudo, quanto aos seus objetivos é exploratória e descritiva. A pesquisa exploratória, do tipo revisão bibliográfica, na qual foram utilizadas fontes como livros, artigos e revistas físicas e digitais, que tratam sobre o tema, bem como em documentação existente na Instituição, objeto do estudo do presente artigo. Seguida de uma pesquisa descritiva, de abordagem qualitativa que, além utilizar fontes como relatórios e demais documentos do setor em questão, deve coletar dados com gestores e funcionários da instituição, numa amostra não probabilística realizada com 5 colaboradores e 2 gestores, em entrevistas de roteiro não estruturado. Os dados da pesquisa serão analisados por meio de tabelas em perspectiva qualitativa.

\section{Fundamentação Teórica}

\subsection{Desenvolvimento Econômico Sustentável}

O conceito de desenvolvimento sustentável manifestou-se mundialmente no relatório de Brundtland em 1987, consequência de análises coordenadas pela Comissão Mundial sobre o meio ambiente e o desenvolvimento, que foi criada em 1983 pela Assembleia Geral das Nações Unidas, com o propósito de desenvolver o crescimento econômico e superar a pobreza dos países desenvolvidos e em desenvolvimento.

Este em muito contribuiu para impor a referência ao desenvolvimento sustentável como novo elemento semântico da linguagem internacional e como elemento de focalização dos trabalhos dos peritos das organizações internacionais (HATEM 1997, GODARD, 1997, p. 110). 
No Brasil, em 1992, com a Rio 92 (ECO 92 ou Cúpula da Terra) o tema foi amplamente discutido, sendo mostrado à humanidade a necessidade de conciliar a responsabilidade social, o desenvolvimento econômico e a aplicação dos recursos naturais disponíveis. Os participantes do evento concluíram que os países desenvolvidos eram os maiores responsáveis pelos danos ao meio ambiente e que os países em desenvolvimento necessitavam de apoio financeiro e tecnológico para caminhar ao novo conceito de sustentabilidade. O fato mais importante é que a partir deste evento ficou concretizado o conceito de Desenvolvimento Sustentável (Kraemer, 2000).

Desde então, a sustentabilidade vem sendo um tema recorrente até mesmo no mercado de valores. Há alguns anos a Bolsa de Valores de São Paulo - BOVESPA- criou o Índice de Sustentabilidade Empresarial - ISE - qualificando empresas que privilegiam os pilares da sustentabilidade: econômico, social e ambiental. A empresa é rigorosamente avaliada e recebe o selo que a qualifica como uma empresa comprometida com a sustentabilidade. O ISE é o indicador utilizado para mensurar o desempenho médio das cotações dos ativos de empresas com reconhecido comprometimento com a sustentabilidade empresarial. O Conselho Deliberativo do ISE seleciona, no máximo, 40 empresas para compor a carteira do índice que receberão os selos (BM\&FBovespa, 2017).

De acordo com Savitz (2007), para uma empresa ser considerada sustentável é preciso que consiga simultaneamente aumentar seus lucros, defender o meio ambiente e melhorar a vida dos stakeholders ${ }^{3}$. Popularmente sustentabilidade é definida como uma forma de substituir um método poluente prejudicial ao meio ambiente por outro menos poluente, mas pode-se afirmar que sustentabilidade em gestão remete a um controle complexo que visa manter a harmonia em critérios ambientais / ecológicos com critérios econômicos e sociais.

\subsection{Marketing Verde ou Marketing Ambiental}

Peattie e Charter (2003) citam que as mudanças na sociedade e no meio ambiente, acabam forçando as empresas a considerar vários grupos interessados na tomada de decisão. Construir relacionamentos com clientes, fornecedores, empregados e comunidade pode ser o eixo central para a competitividade e para formar uma sustentabilidade no desenvolvimento das estratégias.

O marketing verde, conhecido também como marketing ambiental ou marketing ecológico, é uma modalidade derivada do marketing básico que visa atender às necessidades daqueles que possuem um comportamento diferenciado por se preocuparem com medidas sustentáveis e com os danos à natureza. Consiste na aplicação da promoção, produção e também na recuperação de produtos que são ecológicos e sensíveis ao meio ambiente (Kurtz \& Boone, 2001). Muitos acreditam que o marketing verde se resume tão somente à promoção ou publicidade de produtos com atributos ambientais. Alguns termos como produtos recicláveis, reutilizáveis, "amigos da camada de ozônio" e "amigos do meio ambiente" são associados pelos consumidores ao conceito de marketing verde. Embora esses termos estejam associados ao marketing verde, o conceito deste é muito mais amplo, podendo ser aplicado aos bens de consumo, bens de produção e até mesmo aos serviços (Xavier \& Chiconatto, 2013).

Para tanto, é necessário promover uma consciência ambiental através de um desenvolvimento sustentável, consumindo com menos desperdício, reutilizando e reciclando os recursos. A conscientização e a prevenção são essenciais para garantir o desenvolvimento sustentável e diminuir o impacto causado ao meio ambiente e a todas as formas de vida.

${ }^{3}$ Stakeholders: são grupos de interesse que se relacionam, afetam e são afetados por uma instituição e suas atividades (Orchis, Yung e Morales, 2002) 


\subsection{Responsabilidade Social Corporativa}

O termo Responsabilidade Social Corporativa foi utilizado pela primeira vez nos Estados Unidos nos anos 1960, na mesma época que a sociedade passou a exigir uma maior responsabilidade das empresas, solicitando a prestação de contas de suas ações sociais, o que denominamos até hoje de balanço social (Rico, 2004). O termo Responsabilidade Social Corporativa, segundo Carroll (1999), é conceituado por empresas que atuam de acordo com as expectativas que a sociedade possui delas, num dado momento, ao nível das suas ações econômicas, legais, éticas e discricionárias. O tema é interdisciplinar, multidimensional e associado a uma abordagem sistêmica, focada nas relações entre stakeholders diretos e indiretos ao negócio da empresa. Essa nova realidade pode ser definida como o compromisso que uma organização deve ter para com a sociedade, expresso por meio de atos e atitudes que a afetem positivamente, de modo amplo, ou a alguma comunidade, de modo específico, agindo proativamente e coerentemente no que tange a seu papel específico na sociedade e a sua prestação de contas para com ela (Ashley \& Cardoso, 2002). De Araújo e Rosana (2006) afirma, também, que essa perspectiva de ações sociais passa a compor o novo quadro de objetivos das organizações. Sendo assim, a Responsabilidade Social Corporativa resume-se ao comprometimento permanente dos empresários em adotar um comportamento ético e contribuir para o desenvolvimento econômico, melhorando simultaneamente a qualidade de vida de seus empregados e de suas famílias, da comunidade local e da sociedade como um todo.

Com a revolução industrial e o aumento da população, as cidades têm sido afetadas por grandes quantidades de lixo industrial e urbano e que podem ser extremamente ofensivos para o meio ambiente e para o bem-estar da população. Situações iguais a estas geraram no campo da logística uma série de estudos em vários países em função da grande demanda e do rápido crescimento de resíduos e sobras decorrentes de atividades econômicas ligadas à produção e ao consumo (Hofmeister, 2014).

Nas organizações, a responsabilidade legal equivale ao cumprimento das leis e adoção de todas as regulamentações necessárias, e a responsabilidade ética diz respeito às práticas e ações esperadas ou proibidas pela sociedade, relacionadas à honestidade, justiça e direitos (Borges, Miranda \& Valadão, 2007). Contudo, é aceita a ideia de que na atividade empresarial, além das dimensões econômica e legal, exista também a dimensão ética (Machado Filho \& Zylbersztajn, 2004). A Responsabilidade Social Corporativa vem deste modo, como uma forma de obter um relacionamento entre as empresas e a sociedade em geral, estando integrada ao conceito de desenvolvimento sustentável (Back, 2015).

Segundo a Fundação Nacional da Qualidade (FNQ, 2011), para atender ao quesito de responsabilidade socioambiental, a instituição deve implementar processos gerenciais que propiciem a geração de produtos, processos e instalações seguros aos usuários, a população e ao meio ambiente, de forma a fomentar o desenvolvimento social.

\subsection{Gerenciamento de Resíduos de Serviços de Saúde}

Segundo a norma brasileira NBR 10004/04 da Associação Brasileira de Normas Técnicas (ABNT), resíduos sólidos são definidos como resíduos em estado sólido e semissólidos, produtos da ação da indústria, de atividades domésticas, do comércio, de serviços agrícolas e também de serviços hospitalares (ABNT, 2004).

Os Resíduos Sólidos Hospitalares ou, como é mais comumente denominado, lixo hospitalar, podem tornar-se um problema de saúde pública, devido principalmente à falta de informações da população sobre suas particularidades, ocasionando riscos à saúde (Cafure \& Patriarcha-Graciolli, 2014).

Resíduos de Serviços da Saúde (RSS), para as normas brasileiras de terminologia, classificação, manuseio e coleta de resíduos e serviços de saúde, são definidos como resíduos resultantes das atividades exercidas por estabelecimentos prestadores de serviços de saúde, abrangendo os resíduos provenientes das mais diversas fontes potencialmente geradoras, como hospitais, clínicas médicas, veterinárias, odontológicas, 
farmácias, ambulatórios, postos de saúde, laboratório de análises clínicas, laboratórios de análises de alimento, laboratórios de pesquisa, consultórios médicos e odontológicos, empresas de biotecnologia, casas de repouso e casas funerárias (Schneider, Rêgo, Caldart \& Orlandin, 2004).

No Brasil, a fiscalização do gerenciamento de resíduos é feita pelo Conselho de Meio Ambiente CODEMA. O órgão regula e fiscaliza o funcionamento e a legislação da logística reversa e tem como objetivo um controle mais eficaz quanto à natureza da fabricação das embalagens, dos produtos e as suas especificações técnicas. Também é ele que promove a implantação das diretrizes metodológicas que avaliam os impactos ambientais, sociais e econômicos provenientes da adoção da logística reversa e o prazo mínimo para o setor se adequar à nova legislação vigente.

Diante disso, a Diretoria de Vigilância Sanitária - DIVS - e o Conselho Estadual de Meio Ambiente - CONSEMA - aprovaram no final de 2013 a Resolução Conjunta CONSEMA e DIVS nº 01 que:

Estabelece a obrigatoriedade de elaboração e apresentação do Plano de Gerenciamento de Resíduos de Serviço de Saúde - PGRSS e seus documentos complementares atendendo às exigências da resolução da RDC ANVISA $\mathbf{n}^{\mathbf{0}} \mathbf{3 0 6 / 2 0 0 4}$. Os Geradores de resíduos de serviços de saúde (RSS) são todos os serviços relacionados com o atendimento à saúde humana ou animal, inclusive os serviços de assistência domiciliar e de trabalhos de campo; laboratórios analíticos de produtos para saúde; necrotérios, funerárias e serviços onde se realizem atividades de embalsamamento (somatoconservação); serviços de medicina legal; drogarias e farmácias; estabelecimentos de ensino e pesquisa na área de saúde; centros de controle de zoonoses; distribuidores de produtos farmacêuticos, importadores, distribuidores e produtores de materiais e controles para diagnóstico in vitro; unidades móveis de atendimento à saúde; serviços de acupuntura; serviços de tatuagem, dentre outros similares. (Ministério da Saúde - RESOLUÇÃO RDC Nº 306, DE 7 DE DEZEMBRO DE 2004)

O PGRSS deve ser formulado de acordo com as características particulares de cada estabelecimento, seguindo as regulamentações e normas vigentes, tendo que contemplar as alternativas e o gerenciamento viáveis, de recursos indispensáveis e o pessoal necessário e responsável pela sua implementação.

A importância de implantar o gerenciamento ambiental está na possibilidadede correções de pontos de falhas, aumento da qualidade dos serviços prestados, melhor qualidade da saúde pública e ambiental, valorização da imagem da instituição, redução de custos e origem de recursos (Calegare, Godoy \& Silveira, 2006).

\section{Apresentação e Análise de Dados}

O hospital aqui estudado é público, administrado por uma organização social, caracterizado como hospital especializado e com mais de trinta anos de atuação. Desde o início, o atendimento ao cliente foi marcado por forte humanismo por parte de toda a equipe envolvida, e com o crescimento da instituição, foram incorporados novos serviços e exames, tornando a instituição referência no país todo. $\mathrm{O}$ aumento no número de pacientes atendidos e, consequentemente o aumento dos resíduos gerados no ambiente hospitalar, levaram a direção do hospital a se preocupar com os impactos ao meio ambiente do lixo hospitalar gerado e também a se adequarem a legislação vigente.

O Programa de Gerenciamento de Resíduos de Serviços de Saúde do hospital - PGRSS - teve início em 2003 com criação de grupos de trabalho em cada uma de suas unidades e capacitação à distância promovida pelo Ministério da Saúde e Universidade Federal de Santa Catarina sobre o tema "Saúde Ambiental e Gestão de Resíduos de Serviços de Saúde, baseando na Resolução 5 de 1993 do CONAMA. Mas foi em 2006, seguindo o previsto na RDC 33 da ANVISA que continha o regulamento técnico para o gerenciamento de resíduos de serviços de saúde e a partir de um diagnóstico do gerenciamento de resíduos em todas as unidades, com assessoria de uma empresa contratada, que o PGRSS realmente passou a atuar de fato, sendo reconhecido como um requisito legal e de relevância para a instituição. A partir deste ano foram criados grupos de trabalho no Hospital, tendo um coordenador por unidade para que haja uma padronização das ações em toda a rede, há também constantes capacitações dos coordenadores e 
colaboradores. A inserção do PGRSS no Programa de Gestão da Qualidade e no Planejamento Estratégico é realizada por meio de documentos controlados e auditorias e reforma dos abrigos de resíduos, bem como aquisições de lixeiras e containers com especificações baseadas na legislação vigente. $\mathrm{O}$ volume de resíduos aproximados é descrito no quadro 1.

A instituição hospitalar conta com aproximadamente 362 colaboradores (relatório do sistema interno de 2015), e a cada colaborador cabe à obrigatoriedade de atuar com comprometimento no manejo de resíduos seguindo a legislação vigente e o manejo de resíduos. Com relação às atribuições relacionadas ao PGRSS, cabe ao Diretor do Hospital assegurar que os resíduos sejam manuseados de forma a garantir a segurança dos funcionários, dos usuários, da comunidade e do meio ambiente. O Coordenador do PGRSS deve implementar e assegurar a manutenção do PGRSS e a aplicação das legislações e normas técnicas pertinentes, cabendo a este garantir a segurança ocupacional dos trabalhadores envolvidos no gerenciamento, monitorar os riscos existentes no processo, colaborar institucionalmente no gerenciamento dos resíduos do hospital, bem como atuar preventivamente contra agravos relacionadas ao PGRSS e participar da aprovação dos procedimentos de manejo dos resíduos. Cabe a cada responsável de setor conforme Matriz de Autoridade e Responsabilidade ou pessoa designada por este, acompanhar localmente às atividades de geração, segregação e acondicionamento dos resíduos e motivar as ações relacionadas ao PGRSS no setor. Cabe ainda, incluir o PGRSS no site da Vigilância Sanitária e atualizar sempre que necessário conforme a versão em vigor.

Quadro 1 - Volume aproximado de resíduos gerados pela instituição

\begin{tabular}{|l|c|}
\hline GRUPO DE RESÍDUO & $\begin{array}{c}\text { QUANTIDADE } \\
\text { APROXIMADA }\end{array}$ \\
\hline Infectante A1 & $1.950 \mathrm{Kg} / \mathrm{mês}$ \\
\hline Infectante A4 & $785 \mathrm{Kg} / \mathrm{mês}$ \\
\hline Químico & $2 \mathrm{Kg} / \mathrm{mês}$ \\
\hline Pérfuro-cortante & $395 \mathrm{Kg} / \mathrm{mês}$ \\
\hline
\end{tabular}

Fonte: Indicadores da Instituição, 2014.

O PGRSS tem bases científicas, técnicas, normativas e legais, assim não é apenas um documento de consulta, ele tem uma característica dinâmica de apontar melhorias e descrever metas para se alcançar o objetivo final: a preservação do meio ambiente, bem como deve atender aos requisitos da legislação vigente além de contemplar atividades do manejo dos resíduos, educação continuada, saúde do trabalhador. Sendo um compromisso de o hospital desenvolver o conceito e promover a qualidade e atendendo os requisitos legais e dos clientes. O Sistema de Gestão da Qualidade do Hospital envolve todos os processos, assim, o Programa de Gerenciamento de Resíduos também reflete os valores da Instituição e a sua Política de Qualidade.

Os impactos sociais e ambientais de todos os produtos, processos e instalações são verificados e tratados através do acompanhamento do Plano de Gerenciamento de Resíduos de Serviços de Saúde pelo Diretor do Hospital e pelo coordenador do PGRSS do hospital. Através do levantamento de todos os resíduos gerados divididos por classes, são então estabelecidas medidas técnicas para identificação, segregação, acondicionamento e destino final, bem como, realizados treinamentos com os colaboradores para esclarecimento das normas vigentes. Esta prática trata os impactos socioambientais de forma proativa e abrange todos os serviços do hospital. A coleta e destinação final dos resíduos hospitalares são realizadas 
através de um contrato de prestação de serviço com empresa terceirizada, tendo a finalidade de prevenir situações adversas à sociedade e ecossistemas.

Quadro 2 - Práticas de gestão dos critérios de responsabilidade socioambiental x atendimento dos requisitos legais

\begin{tabular}{|c|c|c|}
\hline Critérios & Perguntas & Prática Identificada \\
\hline \multirow{5}{*}{ 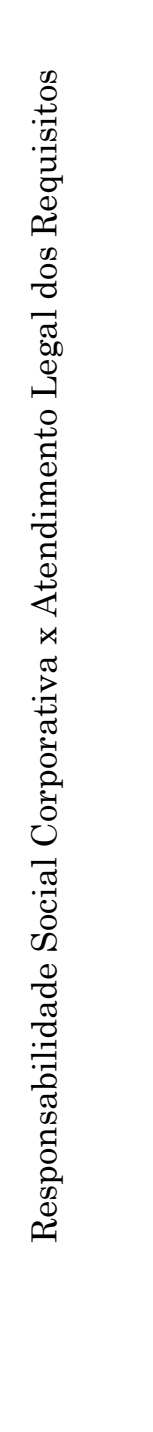 } & $\begin{array}{l}\text { Como são tratados e identificados os } \\
\text { impactos socioambientais negativos } \\
\text { dos produtos, processos e instalações? }\end{array}$ & $\begin{array}{l}\text { PGRSS - Plano de } \\
\text { Gerenciamento de Resíduos de } \\
\text { Serviço de Saúde; Empresa } \\
\text { terceirizada responsável pelo } \\
\text { recolhimento e destinação final } \\
\text { de Resíduos Hospitalares. }\end{array}$ \\
\hline & $\begin{array}{l}\text { Como os impactos socioambientais de } \\
\text { todos os processos envolvidos, e demais } \\
\text { informações relevantes relativas à } \\
\text { responsabilidade da Instituição são } \\
\text { comunicadas a comunidade e ao } \\
\text { público interno? }\end{array}$ & $\begin{array}{l}\text { Plano de Marketing através de } \\
\text { algumas ações como - } \\
\text { elaboração do jornal interno da } \\
\text { instituição, atualização das } \\
\text { redes sociais, planejamento e } \\
\text { execução de campanhas } \\
\text { publicitárias, auditorias do } \\
\text { sistema de gestão da qualidade, } \\
\text { análise dos indicadores e dados } \\
\text { estatísticos, pesquisa de } \\
\text { satisfação do cliente. }\end{array}$ \\
\hline & $\begin{array}{l}\text { Como é realizada a identificação e } \\
\text { análise dos requisitos legais, } \\
\text { regulamentares e implementação de } \\
\text { melhorias? }\end{array}$ & $\begin{array}{l}\text { Órgãos competentes - } \\
\text { Coordenador da comissão do } \\
\text { PGRSS da Instituição; } \\
\text { colaboradores. }\end{array}$ \\
\hline & $\begin{array}{l}\text { Como a Instituição promove ações } \\
\text { voltadas ao desenvolvimento } \\
\text { sustentável? }\end{array}$ & $\begin{array}{l}\text { PGRSS - Programa de } \\
\text { separação dos resíduos } \\
\text { recicláveis; Programa 5S; ISSO } \\
9002 \text { - Integração de novos } \\
\text { colaboradores. }\end{array}$ \\
\hline & $\begin{array}{l}\text { Como são identificadas as } \\
\text { necessidades e expectativas dos } \\
\text { clientes e utilizadas para } \\
\text { implementação de melhorias? }\end{array}$ & $\begin{array}{l}\text { Pesquisa de Satisfação do } \\
\text { Cliente; Programa de Gestão da } \\
\text { Qualidade; Auditorias internas } \\
\text { e externas. }\end{array}$ \\
\hline
\end{tabular}

Fonte: Indicadores da Instituição, 2016

Através da análise do critério de sociedade do PGRSS e as práticas de gestão evidenciadas em conversa com os gestores da instituição, foi possível avaliar se estas práticas são decorrentes de uma RSC ou um atendimento aos requisitos legais, conforme ilustrado no Quadro 3: 
Quadro 3 - Classificação das práticas de gestão

\begin{tabular}{|c|c|c|c|}
\hline Práticas Identificadas & $\begin{array}{l}\text { Requisitos } \\
\text { Legais }\end{array}$ & $\mathrm{RSC}$ & Outros \\
\hline $\begin{array}{l}\text { PGRSS - Plano de Gerenciamento } \\
\text { de Resíduos de Serviço de Saúde }\end{array}$ & $\mathrm{X}$ & & \\
\hline Pesquisa de Satisfação de Clientes & & & $\mathrm{X}$ \\
\hline $\begin{array}{l}\text { Programa 5S; ISO 9002; Auditorias } \\
\text { internas e externas. }\end{array}$ & & $\mathrm{X}$ & \\
\hline Integração de Novos Colaboradores & & $\mathrm{X}$ & \\
\hline $\begin{array}{l}\text { Programa de separação de Resíduos } \\
\text { Recicláveis }\end{array}$ & $\mathrm{X}$ & & \\
\hline $\begin{array}{l}\text { Contrato Terceirizado para } \\
\text { destinação dos Resíduos } \\
\text { Hospitalares }\end{array}$ & $\mathrm{X}$ & & \\
\hline Coordenador do PGRSS & & $\mathrm{X}$ & \\
\hline $\begin{array}{l}\text { Acompanhamento de Normas e } \\
\text { Regulações emitidas pelos Órgãos } \\
\text { Competentes }\end{array}$ & $\mathrm{X}$ & & \\
\hline $\begin{array}{l}\text { Plano de Marketing e Plano de } \\
\text { Gestão da Qualidade }\end{array}$ & & & $\mathrm{X}$ \\
\hline $\begin{array}{l}\text { Descarte de Equipamentos } \\
\text { eletrônicos antigos }\end{array}$ & & $\mathrm{X}$ & \\
\hline $\begin{array}{l}\text { Descarte de resíduos de } \\
\text { hemocomponentes }\end{array}$ & $\mathrm{X}$ & & \\
\hline
\end{tabular}

Fonte: Indicadores da Instituição, 2016

Tendo como base os dados identificados na pesquisa com os gestores e colaboradores, nota-se que além da preocupação em atender os requisitos, existe também como valor institucional que não só é aplicado, mas também idealizado pelos funcionários, e também na política da qualidade com uma melhoria contínua assegurada por meio de uma gestão participativa, cumprindo não só os requisitos legais, mas também o seu papel na sociedade como um todo. É percebido que através das campanhas de marketing realizadas, existe preocupação e interesse na opinião do público atendido pelo hospital, e por meio das auditorias é possível identificar se os procedimentos estão sendo feitos corretamente, identificar e corrigir processos falhos e padronizar as ações da instituição.

A comunicação dos impactos socioambientais relevantes ocorre através do plano de marketing, elaborado e revisado anualmente com o auxílio de uma equipe especializada em marketing própria do 
Hospital. É o plano de marketing que norteia toda política de comunicação interna e externa da instituição, através de ações como a elaboração do jornal interno da instituição e publicação de newsletter, atualização das redes sociais e acompanhamento dos índices, com o planejamento e execução das campanhas publicitárias da instituição, e também através de auditorias do sistema de gestão da qualidade, análise dos indicadores e dados estatísticos, pesquisa de satisfação do cliente e realização de coletas externas.

Os requisitos legais, regulamentares e contratuais aplicáveis ao Hospital são acompanhados permanentemente através da assessoria da empresa gestora do mesmo, que interage de forma disciplinada com a direção do hospital onde dissemina os temas relacionados a cada rede mantida pela instituição. Para a área da saúde todos os requisitos legais e regulamentares são analisados pela gerência técnica e os pareceres enviados para o diretor do hospital. Da mesma maneira a empresa gestora do hospital determina as auditorias internas e externas, visando garantir a execução dos aspectos legais pertinentes à instituição. A gerência técnica realiza junto à gerência administrativa a análise da legislação e normas vigentes à organização (portarias regulamentadoras do Ministério da Saúde, Vigilância Sanitária entre outros) e sempre que necessário promove a discussão das necessidades de adaptação e impacto financeiro junto à direção do hospital, para deliberar as ações a serem implementadas.

As ações relativas ao desenvolvimento da gestão da qualidade são discutidas e promovidas anualmente pela equipe do programa de qualidade, através de práticas de gestão específicas. São realizadas a separação e classificação dos resíduos não hospitalares, em comum, orgânico e reciclável, sendo de responsabilidade da coordenação do PGRSS a fiscalização do mesmo. O Programa 5S, bem como as auditorias internas e realizadas semestralmente, sendo de responsabilidade da comissão da qualidade, e preconizam a otimização de recursos e o aproveitamento dos materiais e equipamentos. As necessidades e expectativas de desenvolvimento da sociedade são identificadas, analisadas através da pesquisa de satisfação de clientes, onde as informações coletadas são utilizadas para a definição e a melhoria da atuação social da instituição.

No quesito social, dentre os projetos realizados pela instituição, podemos citar o projeto de doação de sangue voluntária, onde uma equipe multidisciplinar visita escolas e empresas visando aumentar o número de doações voluntárias e falar da importância de manter os estoques de hemocomponentes da instituição e de suas unidades. No caso das empresas, a instituição disponibiliza transporte para os funcionários doarem sangue no hospital.

\section{Considerações Finais}

Ao realizarmos a pesquisa na instituição, confrontando as práticas identificadas como atendimento aos requisitos exigidos pela legislação e a responsabilidade social corporativa, foi possível observar um equilíbrio entre ambas. Embora as maiorias das práticas ainda sejam em virtude dos requisitos legais, existe uma preocupação da instituição com o bem-estar da sociedade. Com uma análise aprofundada das práticas de gestão, foi somente com a capacitação dos colaboradores no PGRSS da instituição que se iniciou esta preocupação, e reflete a importância que o programa trouxe para toda a rede hospitalar. A RSC também implica em a empresa seja responsável pelos efeitos de suas atividades e bem-estar de seus empregados, clientes, comunidade local e sociedade (Borger, 2001: Machado Filho \& Zylbersztajn, 2004).

Neste estudo, fica evidenciado que o PGRSS da instituição não é apenas um atendimento às normas legais, mas que foi em virtude de atender a legislação que nasceu o interesse nas práticas relacionadas a responsabilidade social corporativa. Nota-se que, além de ter que atender aos requisitos legais em virtude dos resíduos hospitalares gerados, criou-se também uma mentalidade de preocupação com o meio ambiente e com a sociedade como um todo.

O hospital demostra preocupar-se em melhorar sua gestão todos os anos, revisando-a e buscando a excelência nas práticas adotadas, bem como avaliar seus métodos através de auditorias internas e externas e indicadores, tendo como objetivo atuar de forma ética com a sociedade de maneira geral e oferecer um manejo seguro dos resíduos produzidos. 


\section{BIBLIOGRAFÍA}

Associação Brasileira de Normas Técnicas - ABNT (1993). NBR 12808: Resíduos de Serviços de Saúde: classificação. São Paulo.

(2004). NBR 10.004: Classificação de resíduos sólidos: Rio de Janeiro.

Ashley, P. A., \& Cardoso, J. G. (2002). A responsabilidade social nos negócios: um conceito em construção. Ética e responsabilidade social nos negócios, 2.

Back, L. S. (2015). Responsabilidade social corporativa em empresas de pequeno e médio porte: fatores que influenciam a adoção de iniciativas de sustentabilidade.

BM\&FBovespa (2017) Índice de Sustentabilidade Eempresarial ISE: O Índice, 2017. Disponível em: $<$ http://www.bmfbovespa.com.br/pt_br/produtos/indices/indices-de-sustentabilidade/indice-de-sustentabilidadeempresarial-ise.htm> Acessado em: 10 jul. 2017

Borger, F. G. (2001). Responsabilidade social: efeitos da atuação social na dinâmica empresarial (Doctoral dissertation, Universidade de São Paulo).

Borges, J. F., Miranda, R., \& Valadão Júnior, V. M. (2007). O discurso das fundações corporativas: caminhos de uma" nova" filantropia?. Revista de Administração de Empresas, 47(4), 1-15

Cafure, V. A., \& Patriarcha-Graciolli, S. R. (2015). Os resíduos de serviço de saúde e seus impactos ambientais: uma revisão bibliográfica. Interações (Campo Grande), 16(2).

Calegare, L., Godoy, L., \& Silveira, D. (2006). Contribuições do sistema de gerenciamento ambiental (sga) à qualidade dos serviços de saúde hospitalar. SIMPÓSIO INTERNACIONAL DE QUALIDADE AMBIENTAL, 5.

Carroll, A. B. (1979). A three-dimensional conceptual model of corporate performance. Academy of management review, 4(4), 497-505.

de Araújo, M., \& Rosana, M. (2006). Exclusão social e responsabilidade social empresarial. Psicologia em estudo, 11(2).

Fundação Nacional da Qualidade - FNQ (2011). Critérios rumo a excelência Modelo de excelência da gestão. Recuperado em 31 de outubro de 2011 da Fundação Nacional de Qulidade website: <http://www.fnq.org.br/informese/publicacoes/modelo-de-excelencia-da-gestao/criterio-rumo-a-excelencia>.

Irigaray, H. A. R.; Vergara, S. C.; Santos, M. C. F. (2013) Responsabilidade social corporativa: um duplo olhar sobre a Reduc. RAM. Revista de Administração Mackenzie, 14(6).

Hofmeister, L. F. (2014). Logística reversa e gestão de resíduos em organizações hospitalares: os casos do Hospital Santa Cruz e do Hospital Estrela.

Kelm, M. L., da Silva Renz, C. L., Allebrandt, S. L., \& Oneide Sausen, J. (2014). Institucionalização das iniciativas socioambientais das organizações: interfaces entre a teoria do desenvolvimento social de Habermas e o isomorfismo da teoria institucional. Cadernos EBAPE. BR, 12.

Kraemer, M. E. (2000) Responsabilidade social - uma alavanca para a sustentabilidade. Revista Pensar Contábil, Responsabilidade Social e Ambiental, ano 3, número 9. CRC/RJ. Rio de Janeiro.

Kurtz, D. L., \& Boone, L. E. (1992). Contemporary marketing. The Dryden Press.

Machado Filho, C. A. P., \& Zylbersztajn, D. (2004). A empresa socialmente responsável: o debate e as implicações. Revista de Administra\&ccdeil; ão da Universidade de São Paulo, 39(3).

Orchis, M. A., Yung, M. T., \& Morales, S. C. (2002). Impactos da responsabilidade social nos objetivos e estratégias empresariais. Responsabilidade Social das Empresas, 37-70.

Peattie, K., \& Charter, M. (2003). Green marketing. The marketing book, 5, 726-755.

Rico, E. D. M. (2004). A responsabilidade social empresarial e o Estado: uma aliança para o desenvolvimento sustentável. São Paulo em perspectiva, 18(4), 73-82. 
Sanches, C. S. (2000). Gestão ambiental proativa. Revista de Administração de Empresas, 40(1), 76-87.

Savitz, A. W., \& Weber, K. (2007). A empresa sustentável: o verdadeiro sucesso é o lucro com responsabilidade social e ambiental. Elsevier.

Schneider, V. E., Rêgo, R. D. C. E. D., Caldart, V., \& Orlandin, S. M. (2004). Manual de gerenciamento de resíduos sólidos de serviços de saúde. In Manual de gerenciamento de resíduos sólidos de serviços de saúde. 2a ed. revisada e ampliada.

Xavier, R. N., \& Chiconatto, P. (2014). O Rumo do Marketing Verde nas Organizações: Conceito, Oportunidades e Limitações. Revista Capital Científico-Eletrônica (RCCe)-ISSN 2177-4153, 12(1), 133-147. 